postoperative day 19. His 6-month follow-up examination showed no recurrence of the infection.

\section{Discussion}

Mycotic aortic aneurysm is an extremely rare complication of arterial infection, accounting for fewer than $1 \%$ of aortic aneurysm repairs. The bacterial organisms commonly identified include Staphylococcus aureus, Staphylococcus epidermidis, Salmonella species, Streptococcus species, Haemophilus influenzae, Pseudomonas species, Candida species, and Escherichia coli. ${ }^{1}$ Isolation of $S$ pneumoniae in the mycotic aneurysm seems to be rare; we have found only a few reports associated with this pathogen. ${ }^{2,3}$

It has been suggested that in situ insertion of cryopreserved aortic homograft would reduce the postoperative infection rate and improve survival of this group of high-risk patients ${ }^{4}$; however, most patients with mycotic aneurysm require emergency intervention, and access to a tissue bank is not feasible. In certain circumstances, such as active purulent infection by $S$ pneumoniae, homografts have been reported to fail. ${ }^{3}$

How to deal with the ILVA is debated. We decided to preserve our patient's ILVA for two reasons. One was that in certain aortic arch anomalies the left common carotid artery does not supply normal blood flow, and the ILVA compensates for this. ${ }^{5}$ Another was that if the vertebrobasilar axis is a dominant left axis or if communication at the arterial circle of Willis is poor because of another arterial lesion, elimination of the ILVA can cause ischemia of the brainstem and cerebellum. The approaches to ILVA reconstruction include en bloc reconstruction of the arch branches, direct anastomosis to the graft branch corresponding to the LSA, and direct anastomosis to the native LSA. ${ }^{6,7}$ Although there are no published long-term results concerning the patency of the reconstructed ILVA, we used a saphenous vein graft interposed between the ILVA and graft branch.

\section{References}

1. Svensson LG, Crawford ES. Cardiovascular and vascular disease of the aorta. Philadelphia: Saunders; 1996.

2. Nijs A, Vandekerkhof J, Cartuyvels R, Magerman K, Mewis A, Peeters $\mathrm{V}$, et al. Streptococcus pneumoniae-infected aneurysm extending from a persistent lobar pneumonia: case report and review of the literature. Eur J Clin Microbiol Infect Dis. 2002;21:389-92.

3. Berdat PA, Malinverni R, Kipfer B, Carrel TP. Homograft failure in mycotic aortic aneurysm caused by Streptococcus pneumoniae. Ann Thorac Surg. 1998;66:1818-9.

4. Vogt PR, Brunner-La Rocca HP, Carrel T, von Segesser LK, Ruef C, Debatin J, et al. Cryopreserved arterial allografts in the treatment of major vascular infection: a comparison with conventional surgical techniques. J Thorac Cardiovasc Surg. 1998;116:965-72.

5. Ad N, Lee P, Cox JL. Type A aortic dissection with associated anomaly of the carotid and vertebral vessels. J Thorac Cardiovasc Surg. 2002; 123:570-1.

6. Kazui T, Washiyama N, Muhammad BA, Terada H, Yamashita K, Takinami M. Improved results of atherosclerotic arch aneurysm operations with a refined technique. J Thorac Cardiovasc Surg. 2001;121: 491-9.

7. Nonami Y, Tomosawa N, Nishida K, Nawata S. Dissecting aortic aneurysm involving an anomalous right subclavian artery and isolated left vertebral artery: case report and review of the literature. $J$ Cardiovasc Surg (Torino). 1998;39:743-6.

\title{
Successful LVAS and RVAS-ECMO support in a patient with fulminant myocarditis who failed to recover from ventricular fibrillation with PCPS and IABP
}

\author{
Satoshi Gojo, ${ }^{a}$ Shunei Kyo, ${ }^{\mathrm{a}, \mathrm{b}}$ Hiroshige Sato, ${ }^{\mathrm{a}}$ Motonobu Nishimura, ${ }^{\mathrm{b}}$ Toshihisa Asakura, ${ }^{\mathrm{a}}$ Hiroyuki Ito, ${ }^{\mathrm{c}}$ and

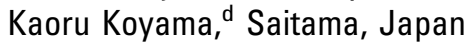

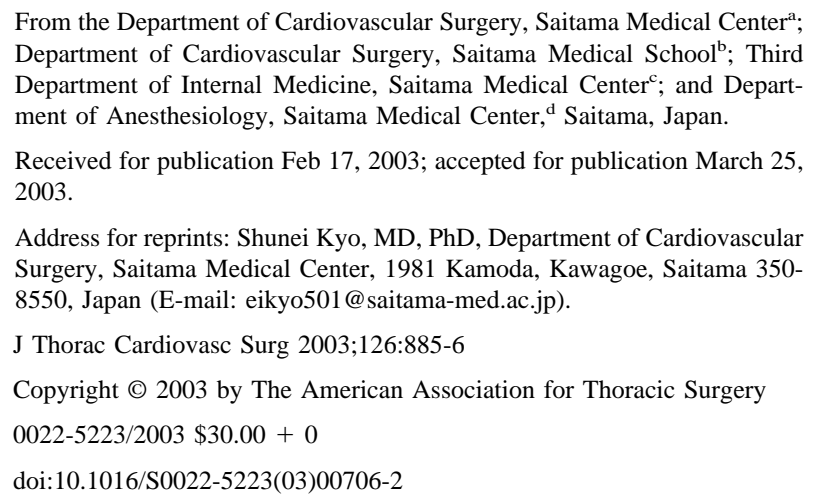

From the Department of Cardiovascular Surgery, Saitama Medical Center ${ }^{\mathrm{a}}$ Department of Cardiovascular Surgery, Saitama Medical School ${ }^{\text {b }}$; Third Department of Internal Medicine, Saitama Medical Centerc; and Department of Anesthesiology, Saitama Medical Center, ${ }^{\mathrm{d}}$ Saitama, Japan.

Received for publication Feb 17, 2003; accepted for publication March 25, 2003.

Address for reprints: Shunei Kyo, MD, PhD, Department of Cardiovascular Surgery, Saitama Medical Center, 1981 Kamoda, Kawagoe, Saitama 3508550, Japan (E-mail: eikyo501@ saitama-med.ac.jp).

J Thorac Cardiovasc Surg 2003;126:885-6

Copyright $\odot 2003$ by The American Association for Thoracic Surgery $0022-5223 / 2003 \$ 30.00+0$

doi:10.1016/S0022-5223(03)00706-2

$\mathrm{F}$ ulminant myocarditis can cause sudden circulatory collapse due to lethal arrhythmia, which is resistant to conventional treatments such as percutaneous cardiopulmonary support (PCPS) and intra-aortic balloon pumping (IABP). We describe the case of a young patient with fulminant end-stage myocarditis who was unable to recover from ventricular fibrillated (Vf) cardiac condition even with PCPS and IABP and who achieved recovery using a left ventricular assist system (LVAS) and a right ventricular assist system (RVAS) with extracorporeal membrane oxygenation (ECMO).

\section{Clinical Summary}

Myocarditis remains a poorly characterized disorder, and it is sometimes difficult to make an early diagnosis. ${ }^{1}$ Although patients 
with fulminant myocarditis (FM) have a better long-term prognosis than those with acute (nonfulminant) myocarditis, ${ }^{2}$ rapid progressive cardiac decompensation in FM soon after diagnosis may be obstinate. The usefulness of PCPS in treating patients with myocarditis who develop cardiogenic shock has been documented. ${ }^{3}$ In cases of exacerbation of multiple organ failure, low flow of PCPS, long-term usage of PCPS, trouble with PCPS cannula, and sustained Vf, the implantation of a ventricular assist system (VAS) is indicated. ${ }^{4}$

\section{Case Report}

A 20-year-old, previously healthy woman (weight: $50 \mathrm{~kg}$; body surface area: $1.46 \mathrm{~m}^{2}$ ), who had a spiking fever of $40^{\circ} \mathrm{C}$ and chest pain, was transferred to our institution from a local hospital. The following morning the patient developed Vf requiring mechanical ventilation, PCPS, and IABP. Despite high doses of inotropic drugs, the patient's systolic blood pressure decreased progressively to $50 \mathrm{~mm} \mathrm{Hg}$ and Vf was still refractory to nifekalant, amiodarone hydrochloride, and DC cardioversion. The decision was made to implant a Toyobo LVAS (Toyobo Inc, Osaka, Japan).

A cardiac muscle sample was taken from the apex, into which the inflow cannula of the LVAS was inserted. Pathological examination revealed that lymphocytes, eosinophiles, and plasma cells had infiltrated severely and a large portion of the perivascular area had suffered myxoid change. The LVAS flow was 2.5 to $3.0 \mathrm{~L} / \mathrm{min}$ at an adequate setting. As ventricular tachycardia (VT) developed at the time of chest closure and was sustained until the following day, RVAS was established using a centrifugal pump (TERUMO Inc, Tokyo, Japan). The outflow and inflow cannulae for RVAS were placed in the main pulmonary artery through the left chest wall and right femoral vein to the right atrium, respectively. Sustained VT ceased a few minutes after RVAS was established. The patient's hemodynamic status improved, with an LVAS flow of 4.0 to $4.5 \mathrm{~L} / \mathrm{min}$.

The RVAS was explanted on day 5 after RVAS implantation. Transthoracic echocardiography (TTE) showed that left ventricular function had recovered well, with an ejection fraction of $62 \%$ at 6 days after LVAS implantation. On day 13 after LVAS implantation, cardiac catheterization was performed with the LVAS off for 30 minutes. However, the patient's cardiac index, determined by Swan-Ganz catheter, was $1.9 \mathrm{~L} / \mathrm{min}$, and tricuspid regurgitation (TR) of grade IV ensued. After the on-off test, the right heart failure deteriorated again. Because mechanical ventilation did not improve arterial blood gas data, RVAS-ECMO was instituted. The TR improved steadily to grade II and the patient's respiratory function recuperated. ECMO was removed on day 5 after RVAS-ECMO implantation. Thereafter, TTE showed that the patient was maintaining good left ventricular function, and the LVAS was removed and tricuspid annuloplasty was performed on day 26 after BVAS implantation. She was transferred to the department of rehabilitation 2 months after explantation.

\section{Comments}

Both the diagnosis and the treatment of myocarditis are problematic. Invasive approaches including VAS appear to be highly beneficial in patients with FM. Cardiac transplantation was avoided in more than half of the patients receiving VAS.

When a patient using an LVAS for severe heart failure is compromised with respiratory failure due to right heart failure, BVAS might not be sufficient to treat such a condition. Because it is disadvantageous to maintain the assist flow of BVAS and intricate to add ECMO with femoral venoarterial bypass to BVAS, we hypothesized that a centrifugal pump combined with ECMO could be utilized to support both right ventricular function and oxygenation. ${ }^{5}$ The decision to place the outflow cannula into either the main pulmonary artery or the left atrium depends on whether a pulmonary hemorrhage is concomitant, recognizing that, in the latter case, there is the risk of sending air into the body. This case report shows that this experimental strategy is justified and functions well in the clinical arena. A centrifugal pump can be placed easily and its versatility, particularly the ease with which ECMO can be added, makes it preferable to pulsatile RVAS as a rapid treatment for FM.

In certain patients with idiopathic dilated cardiomyopathy, the heart may recover its function well while on VAS support. ${ }^{6}$ However, the regained cardiac function is not always long-lasting. On the other hand, recurrence of heart failure is rarely found in cases of $\mathrm{FM}^{2}$ Extracorporeal VAS, rather than intracorporeal VAS, should be a standard method of treatment for FM because of the ease of removing the VAS and due to the strong likelihood of bridging to recovery in such cases.

We would like to express sincere thanks to T. Morita, S. Sasagawa, M. Ishii, and T. Kitawaki for providing expert technical assistance for VAS throughout the treatment.

\section{References}

1. Feldman AM, McNamara D. Myocarditis. N Engl J Med. 2000;343: 1388-98.

2. McCarthy RE III, Boehmer JP, Hruban RH, et al. Long-term outcome of fulminant myocarditis as compared with acute (nonfulminant) myocarditis. N Engl J Med. 2000;342:690-5.

3. Kato S, Morimoto S, Hiramitsu S, Nomura M, Ito T, Hishida H. Use of percutaneous cardiopulmonary support of patients with fulminant myocarditis and cardiogenic shock for improving prognosis. Am J Cardiol. 1999;83:623-5.

4. Acker MA. Mechanical circulatory support for patients with acutefulminant myocarditis. Ann Thorac Surg. 2001;71:S73-6.

5. Fukushima Y, Kyo S, Omoto R. Comparison of veno-arterial bypass with veno-left atrial bypass and veno-pulmonary artery bypass for ECMO support in a swine respiratory failure model. Jap J Artif Organs. 1999;28:284-90.

6. Hetzer R, Muller J, Weng Y, Wallukat G, Spiegelsberger S, Loebe M. Cardiac recovery in dilated cardiomyopathy by unloading with a left ventricular assist device. Ann Thorac Surg. 1999;68:742-9. 